\begin{tabular}{|c|l|}
\hline Title & Existence of infinitely many zero-energy states in a model of supersymmetric quantum mechanics \\
\hline Author(s) & A rai, A sao \\
\hline Citation & Journal of Mathematical Physics, 30(5), 116411170 \\
\hline https://doi.org/10.1063/1.528337 \\
\hline Issue Date & 1989-05 \\
\hline Doc URL & http://hdl.handle.net/2115/13681 \\
\hline Rights & Copyright $\odot 1989$ A merican Institute of Physics \\
\hline Type & article \\
\hline File Information & jmp30-5.pdf \\
\hline
\end{tabular}

Instructions for use 


\title{
Existence of infinitely many zero-energy states in a model of supersymmetric quantum mechanics
}

\author{
Asao Arai \\ Department of Mathematics, Hokkaido University, Sapporo 060, Japan
}

(Received 9 September 1988; accepted for publication 21 December 1988)

\begin{abstract}
The general framework of the $N=2$ Wess-Zumino holomorphic supersymmetric quantum mechanics with polynomial superpotentials is extended to the case of nonpolynomial superpotentials $V(z)(z \in \mathbb{C})$ in a mathematically rigorous way. It is also proved that there exist no fermionic zero-energy states. Under some conditions for $V$, the operator domain of the supercharges and the supersymmetric Hamiltonian are identified. As an example, the model with $V(z)=\lambda e^{\alpha z}(\lambda \in \mathbb{C} \backslash\{0\}, \alpha>0)$ is analyzed in view of index theory. The following remarkable result is proved: There exist infinitely many bosonic zero-energy states which are localized in the momentum space dual to the $\operatorname{Im} z$ direction. The results are applied to two models in atomic and nuclear physics.
\end{abstract}

\section{INTRODUCTION}

In Ref. 1, Jaffe et al. considered two models of supersymmetric quantum mechanics (SSQM), which are the quantum mechanics versions of the two-dimensional, $N=1$ and $N=2$ Wess-Zumino quantum field models; they also computed the Witten index ( $\equiv$ the number of bosonic zeroenergy states minus the number of fermionic zero-energy states) in each model. In particular, it was proved that in the $N=2$ Wess-Zumino SSQM with an arbitrary polynomial superpotential $V(z)(z \in \mathbb{C})$, there exist no fermionic zeroenergy states (the "vanishing theorem") and the Witten in$\operatorname{dex} I_{\mathrm{w}}$ is equal to the number of bosonic zero-energy states, with

$$
I_{\mathrm{w}}=\operatorname{deg} V-1 \text {. }
$$

In this paper, we consider the $N=2$ Wess-Zumino SSQM with nonpolynomial holomorphic superpotentials and try to extend the results for the case of the polynomial potentials considered in Ref. 1. This is at least mathematically interesting: Formula (1.1) shows that the Witten index is determined by the order of singularity of the superpotential at $z=\infty$ and suggests formally that the Witten index is infinite in the case of nonpolynomial holomorphic superpotentials, for they have the essential singularity at $z=\infty$. Note, also, that a nonpolynomial entire function is the limit of a sequence $\left\{V_{n}(z)\right\}_{n=1}^{\infty}$ of polynomials with deg $V_{n}=n$.

In Sec. II, we describe in a mathematically rigorous way a fundamental framework for the Wess-Zumino holomorphic SSQM with not necessarily polynomial superpotentials. We shall show that some results in Ref. 1 can be extended. For example, the "vanishing theorem" holds also in the present case (Proposition 2.5). In Sec. III, we consider the case with $V(z)=\lambda e^{\alpha z}(\lambda \in \mathbb{C} \backslash\{0\}, \alpha>0)$ and prove by identifying the space of the bosonic zero-energy states exactly that there exist infinitely many bosonic zero-energy states. Thus as far as this special model is concerned, the result justifies the above formal argument. It is noted that every bosonic zero-energy state is localized in the momentum space dual to the Im $z$ direction. In Sec. IV, we apply the result in Sec. III to two models in atomic and nuclear physics: One is a model of a nonrelativistic spin- $\frac{1}{2}$ particle in an external SU(2) gauge field and the other is a model of a nonrelativistic nu- cleon in a pion field. These models were discussed in Ref. 1 in order to give physical interpretation to the Wess-Zumino holomorphic SSQM. In each model, the potential is twodimensional and periodic in one direction (e.g., the $y$ direction ). It is shown that each model has infinitely many zeroenergy states which are localized in the momentum space dual to the $y$ direction.

\section{WESS-ZUMINO SSQM WITH GENERAL HOLOMORPHIC SUPERPOTENTIALS}

In this section we recapitulate the definition of the $N=2$ Wess-Zumino holomorphic SSQM ${ }^{1}$ (cf., also, Refs. 2 and 3 ) and extend some mathematical results obtained in the case of polynomial potentials ${ }^{1}$ to the case of general holomorphic potentials.

The Hilbert space $\mathscr{H}$ of state vectors for the model is given by

$$
\mathscr{H}=L^{2}\left(\mathbb{R}^{2} ; \mathbb{C}^{4}\right) \text {. }
$$

In order to define the supercharges, we introduce $4 \times 4$ matrices $\psi_{1}$ and $\psi_{2}$ by

$$
\begin{aligned}
& \psi_{1}=\frac{1}{2}\left(\begin{array}{cc}
0 & I+\sigma_{3} \\
I-\sigma_{3} & 0
\end{array}\right), \\
& \psi_{2}=\frac{1}{2}\left(\begin{array}{cc}
0 & i \sigma_{1}+\sigma_{2} \\
-i \sigma_{1}-\sigma_{2} & 0
\end{array}\right),
\end{aligned}
$$

where $\sigma_{j}, j=1,2,3$ are the Pauli matrices

$\sigma_{1}=\left(\begin{array}{ll}0 & 1 \\ 1 & 0\end{array}\right), \quad \sigma_{2}=\left(\begin{array}{cc}0 & -i \\ i & 0\end{array}\right), \quad \sigma_{3}=\left(\begin{array}{cc}1 & 0 \\ 0 & -1\end{array}\right)$

and $I$ is the $2 \times 2$ identity matrix. The matrices $\psi_{1}$ and $\psi_{2}$ satisfy the anticommutation relations

$$
\begin{aligned}
& \left\{\psi_{j}, \psi_{k}^{*}\right\}=\delta_{j k}, \\
& \left\{\psi_{j}, \psi_{k}\right\}=0, \quad j, k=1,2,
\end{aligned}
$$

where $\{A, B\} \equiv A B+B A$.

Let $V(z)$ be a holomorphic function on $\mathbb{C}$ (not necessarily polynomial) and consider the operators

$$
\begin{aligned}
& Q_{1}=i\left(\psi_{2} \bar{\partial}+\psi_{2}^{*} \partial\right)+i\left\{\psi_{1}(\partial V)-\psi_{1}^{*}(\partial V)^{*}\right\}, \\
& Q_{2}=\psi_{2} \bar{\partial}-\psi_{2}^{*} \partial+\psi_{1}(\partial V)+\psi_{1}^{*}(\partial V)^{*},
\end{aligned}
$$

where $\partial=\partial / \partial z$ and $\bar{\partial}=\partial / \partial z^{*}$ [ the operators $Q_{j}$ given by 
(2.6) and (2.7) are different from those in Ref. 1]. We shall use the usual identification of $\mathbb{C}$ with $\mathbb{R}^{2}$ through the correspondence $z=x+i y \in \mathbb{C} \leftrightarrow(x, y) \in \mathbb{R}^{2}$. Then $Q_{1}$ and $Q_{2}$ can be considered as operators acting in $\mathscr{H}$ given by (2.1). We put

$$
D=C_{0}^{\infty}\left(\mathbb{R}^{2} ; \mathbb{C}^{4}\right) .
$$

Proposition 2.1: The operators $Q_{1}$ and $Q_{2}$ are essentially self-adjoint on $D$. Further, every power of $Q_{1}$ (respectively, $Q_{2}$ ) is essentially self-adjoint on $D$.

Remark: In the case of polynomial potentials $V$, Proposition 2.1 was proved for $Q_{1}$ and $Q_{1}^{2}$ (Ref. 1).

Proof: It is obvious that $Q_{j}$ is symmetric on $D$. We write it as

$$
Q_{1}=-i L
$$

on $D$, where

$$
L=A_{1} \frac{\partial}{\partial x}+A_{2} \frac{\partial}{\partial y}+B(x, y),
$$

with

$$
\begin{aligned}
& A_{1}=-\frac{1}{2}\left(\psi_{2}+\psi_{2}^{*}\right), \quad A_{2}=(i / 2)\left(\psi_{2}^{*}-\psi_{2}\right), \\
& B(x, y)=\psi_{1}^{*}(\partial V(z))^{*}-\psi_{1} \partial V(z) .
\end{aligned}
$$

The operator $L$ is of the form of the first-order differential operators considered in Ref. 4 because $A_{j} ; j=1,2$; and $B$ are $C^{\infty} 4 \times 4$ matrix-valued functions. Note that $A_{1}$ and $A_{2}$ are constant matrices. Hence the "velocity of propagation" (Ref. 4) associated to $L$ is constant. Then a direct application of Ref. 4, Theorem 2.2 gives the desired result on $Q_{1}$. The proof for the case of $Q_{2}$ is quite similar.

Remark: Note that $\left\{A_{j}, A_{k}\right\}=\delta_{j k} / 2, j, k=1,2$. Hence $Q_{1}$ is a Dirac-type operator. The same holds for $Q_{2}$.

We shall denote the closure of $Q_{j} \uparrow D$ by $\bar{Q}_{j}$. Then we have the following lemma.

\section{Lemma 2.2:}

$\bar{Q}_{1}^{2}=\bar{Q}_{2}^{2}$.

Proof: Direct computations give

$$
Q_{1}^{2} \Psi=Q_{2}^{2} \Psi
$$

for all $\Psi$ in $D$. Then, Proposition 2.1 implies (2.9).

We define the non-negative self-adjoint operator $H$ by

$$
H \equiv \bar{Q}_{1}^{2}=\bar{Q}_{2}^{2} \text {. }
$$

Then we have

$$
H^{1 / 2}=\left|\bar{Q}_{1}\right|=\left|\bar{Q}_{2}\right|
$$

and hence, in particular,

$$
D\left(\bar{Q}_{1}\right)=D\left(\bar{Q}_{2}\right)=D\left(H^{1 / 2}\right),
$$

where $D(A)$ denotes the operator domain of operator $A$.

Lemma 2.3: Each $Q_{j}$ maps $D$ into itself and the anticommutation relation

$$
\left\{Q_{1}, Q_{2}\right\} \Psi=0, \quad \Psi \in D
$$

holds. Further, we have

$$
\left(\bar{Q}_{1} \Psi, \bar{Q}_{2} \Phi\right)+\left(\bar{Q}_{2} \Psi, \bar{Q}_{1} \Phi\right)=0, \quad \Phi, \Psi \in D\left(H^{1 / 2}\right) .
$$

Proof: Equation (2.13) follows from direct computations. By a limiting argument using Proposition 2.1, one can extend (2.13) in the form of (2.14).
Let
$N_{\mathrm{F}}=\left(\begin{array}{cc}I & 0 \\ 0 & -I\end{array}\right)$.

Lemma 2.4: For each $j=1,2, N_{\mathrm{F}}$ maps $D\left(\bar{Q}_{j}\right)$ into itself and the anticommutation relations

$$
\left\{N_{\mathrm{F}}, \bar{Q}_{j}\right\} \Psi=0, \quad \Psi \in D\left(\bar{Q}_{j}\right), \quad j=1,2
$$
hold.

Proof: We first prove (2.16) for $\Psi$ in $D$. Then, a limiting argument using Proposition 2.1 gives the desired result.

The Hilbert space $\mathscr{H}$ given by (2.1) has the following orthogonal decomposition:

$$
\mathscr{H}=\mathscr{H}_{+} \oplus \mathscr{H}_{-},
$$

with

$$
\begin{aligned}
& \mathscr{H}_{+}=\left\{\left(\begin{array}{c}
f_{1} \\
f_{2} \\
0 \\
0
\end{array}\right) \mid f_{1}, f_{2} \in L^{2}\left(\mathbb{R}^{2}\right)\right\}, \\
& \mathscr{H}_{-}=\left\{\left(\begin{array}{l}
0 \\
0 \\
f_{1} \\
f_{2}
\end{array}\right) \mid f_{1}, f_{2} \in L^{2}\left(\mathbb{R}^{2}\right)\right\} .
\end{aligned}
$$

Obviously, we have

$N_{\mathrm{F}} \Psi_{ \pm}= \pm \Psi_{ \pm}, \quad \Psi_{ \pm} \in \mathscr{H}_{ \pm}$.

In summary, we have proved that the quadruple $\left\{\mathscr{H},\left\{\bar{Q}_{1}, \bar{Q}_{2}\right\}, H, N_{\mathrm{F}}\right\}$ is a SSQT with $N=2$ supersymmetry in the sense of Ref. 5 (cf., also, Ref. 6); the operators $\bar{Q}_{1}$ and $\bar{Q}_{2}$ are the self-adjoint supercharges, $H$ is the supersymmetric Hamiltonian, and $N_{\mathrm{F}}$ is the fermion number operator. The closed subspace $\mathscr{H}_{+}$(respectively, $\mathscr{H}_{-}$) is the Hilbert space consisting of bosonic (respectively, fermionic) states.

On the domain $D, H$ is explicitly given as

$H=-\overline{\partial \partial}-\psi_{1}^{*} \psi_{2}\left(\partial^{2} V\right)^{*}-\psi_{2}^{*} \psi_{1} \partial^{2} V+|\partial V|^{2}$.

By a general fact of a SSQT, $H$ is reduced by $\mathscr{H}_{ \pm}$; we shall denote the reduced part of $H$ to $\mathscr{H}_{ \pm}$by $H_{ \pm}$. We have

$$
H_{+}=H_{-}+\left(\begin{array}{cc}
0 & -i\left(\partial^{2} V\right) \\
i\left(\partial^{2} V\right)^{*} & 0
\end{array}\right)
$$

and

$$
H_{-}=\left(-\partial \bar{\partial}+|\partial V|^{2}\right)\left(\begin{array}{ll}
1 & 0 \\
0 & 1
\end{array}\right)
$$

on $D$, where we identify $\mathscr{C}_{ \pm}$with $L^{2}\left(\mathbb{R}^{2} ; \mathbb{C}^{2}\right)$.

We now proceed to the index problem. The Witten in$\operatorname{dex} I_{\mathrm{w}}$ is defined by the number of bosonic zero-energy states minus the number of fermionic zero-energy states ${ }^{3}$ :

$$
I_{\mathrm{w}}=n_{+}-n_{-},
$$

with

$$
n_{ \pm}=\operatorname{dim} \operatorname{Ker} H_{ \pm} \text {. }
$$

By Lemma 2.4 and (2.20), for each $j=1,2$ there exists a unique closed linear operator $Q_{j+}: \mathscr{H}_{+} \rightarrow \mathscr{H}_{-}$such that

$$
\bar{Q}_{j}=\left(\begin{array}{cc}
0 & Q_{j+}^{*} \\
Q_{j+} & 0
\end{array}\right) .
$$

It follows from (2.10) that 


$$
H_{+}=Q_{j+}^{*} Q_{j+}, \quad H_{-}=Q_{j+} Q_{j+}^{*}, \quad j=1,2,
$$

which imply that

$$
n_{+}=\operatorname{dim} \operatorname{Ker} Q_{j_{+}}, \quad n_{-}=\operatorname{dim} \operatorname{Ker} Q_{j+}^{*}, \quad j=1,2 .
$$

Hence we obtain

$$
\begin{aligned}
I_{\mathrm{w}} & =\operatorname{dim} \operatorname{Ker} Q_{j+}-\operatorname{dim} \operatorname{Ker} Q_{j+}^{*} \\
& \equiv \text { index } Q_{j+}, \quad j=1,2 .
\end{aligned}
$$

Remark: The arguments leading to (2.26)-(2.29) apply to every SSQT and the results are well known.

Proposition 2.5 (Vanishing theorem): There exist no fermionic zero-energy states:

$$
n_{-}=0 \text {. }
$$

Remark: This result of (2.30) has been established in the case where $V(z)$ is a polynomial (Ref. 1, Proposition 6). In the case where $V$ is not necessarily a polynomial, it may happen that $H_{-}$is not closed on $D(\bar{\partial}) \cap D\left(|\partial V|^{2}\right)$ and hence that $H_{-} \Omega=0$ is not equivalent to $-\partial \bar{\partial} \Omega=0$ and $|\partial V|^{2} \Omega=0$, as in the case of polynomial potentials $V$.

Proof: The operator $h \equiv-\partial \bar{\partial}+|\partial V|^{2}$ is a two-dimensional Schrödinger operator with a non-negative potential. By Proposition 2.1, $C_{0}^{2}\left(\mathbb{R}^{2}\right)$ is a core for $h$. Thus we can apply Lemma A1 in the Appendix to obtain the desired result.

We next consider conditions for a vector to be in Ker $H_{+}=\operatorname{Ker} Q_{j+}$ [see (2.25), (2.27), and (2.28)].

Formulas (2.28) and (2.29) show that as far as the index problem is concerned, it is sufficient to consider one of $\bar{Q}_{j}, j=1,2$. Henceforth we write

$$
Q=\bar{Q}_{1}, \quad Q_{+}=Q_{1+} .
$$

Lemma 2.6: Suppose that $D\left(Q_{+}\right)=D(\partial) \cap D(|\partial V|)$. Then the following hold.

(i) Every vector $(f, g)$ in $\operatorname{Ker} Q_{+}$satisfies

$$
\begin{aligned}
& \left(-\bar{\partial}+|\partial V|^{2}\right) f+\left(\partial^{2} V\right)(\partial V)^{-1} \bar{\partial} f=0, \\
& \left(-\bar{\partial}+|\partial V|^{2}\right) g+\left(\partial^{2} V\right)^{*}(\partial V)^{*-1} \partial g=0
\end{aligned}
$$

in the generalized sense.

(ii) Let $f \in L^{2}\left(\mathbb{R}^{2}\right)$ be a vector satisfying (2.32) in the generalized sense. Then $(f, g)$ is in $\operatorname{Ker} Q_{+}$if and only if $f$ is in $D(\partial) \cap D(|\partial V|)$ and $(\partial V)^{-1} \bar{\partial} f$ is in $L^{2}\left(\mathbb{R}^{2}\right)$, with

$$
g=i(\partial V)^{-1} \bar{\partial} f \text {. }
$$

(iii) Let $g \in L^{2}\left(\mathbb{R}^{2}\right)$ be a vector satisfying (2.33) in the generalized sense. Then $(f, g)$ is in $\operatorname{Ker} Q_{+}$if and only if $g$ is in $D(\partial) \cap D(|\partial V|)$ and $(\partial V)^{*-1} \partial g$ is in $L^{2}\left(\mathbb{R}^{2}\right)$, with

$$
f=-i(\partial V)^{*-1} \partial g \text {. }
$$

Remark: Lemma 2.6 is an elaborate and extended version of Ref. 1, Lemma 8.

Proof: (i) By (2.2), (2.3), (2.6), and the assumption $D\left(Q_{+}\right)=D(\partial) \cap D(|\partial V|)$, we have

$$
Q_{+}=\left(\begin{array}{cc}
-i(\partial V)^{*} & \partial \\
\bar{\partial} & i \partial V
\end{array}\right)
$$

on $D\left(Q_{+}\right)$. Hence every vector $(f, g)$ is in $\operatorname{Ker} Q_{+}$if and only if $(f, g)$ is in $D\left(Q_{+}\right)$and satisfies

$$
\partial g-i(\partial V) * f=0
$$

and

$$
\bar{\partial} f+i(\partial V) g=0 .
$$

Equation (2.37) and the condition $f \in D(\bar{\partial})$ imply that $\bar{\partial} \partial g$ exists as an $L_{\mathrm{loc}}^{1}$ function with

$$
\bar{\partial} \partial g-i\left(\partial^{2} V\right) * f-i(\partial V) * \bar{\partial} f=0 .
$$

It follows from (2.37) and (2.38) that

$$
f=-i(\partial V)^{*-1} \partial g, \quad \bar{\partial} f=-i(\partial V) g .
$$

Substituting these relations into (2.39), we obtain (2.33). Similarly, using (2.38), we can show that $(2.32)$ holds.

(ii) The part "only if " is obvious by (2.38). To prove the part "if," we first note that (2.34) gives (2.38). Hence it follows that $(\partial V) g$ is in $L^{2}\left(\mathbb{R}^{2}\right)$ [i.e., $\left.g \in D(\partial V)\right]$ and

$$
\partial \bar{\partial} f+i\left(\partial^{2} V\right) g+i(\partial V) \partial g=0 .
$$

Using (2.32) and (2.38) to rewrite (2.40), we obtain (2.37). In particular, we have $g \in D(\partial)$. Thus we have proved that $(f, g)$ is in $D(\partial) \cap D(|\partial V|)$ and satisfies (2.37) and (2.38). Therefore $(f, g)$ is in $\operatorname{Ker} Q_{+}$.

(iii) Similar to the proof of (ii).

Finally, we consider conditions under which the assumption $D\left(Q_{+}\right)=D(\partial) \cap D(\partial V)$ in Lemma 2.6 holds.

Lemma 2.7: Suppose that there exists a constant $r>0$ such that for all $z \in \mathbb{C}$ satisfying $\left|\partial^{2} V(z)\right| \geqslant r$, the estimate

$$
\left|\partial^{2} V(z)\right|^{2} \leqslant a|\partial V(z)|^{4}+b
$$

holds with the constants $0<a<1$ and $b \geqslant 0$. Then $D\left(H_{-}\right)=D(\bar{\partial} \bar{\partial}) \cap D\left(|\partial V|^{2}\right)$ and $(2.23)$ holds as an operator equality.

Proof: Let $f$ be in $C_{0}^{\infty}\left(\mathbb{R}^{2} ; \mathbb{C}^{2}\right)$. Then we have

$$
\begin{aligned}
\left\|H_{-} f\right\|^{2}= & \|\partial \bar{\partial} f\|^{2}+\left\|(\partial V)^{2} f\right\|^{2} \\
& -2 \operatorname{Re}\left(\partial \bar{\partial} f,|\partial V|^{2} f\right) .
\end{aligned}
$$

Via integration by parts, one can see that

$$
\begin{aligned}
2 \operatorname{Re}\left(\partial \bar{\partial} f,|\partial V|^{2} f\right)= & \left\|\left(\partial^{2} V\right) f\right\|^{2} \\
& -\|(\partial f)(\partial V)\|^{2}-\|(\bar{\partial} f)(\partial V)\|^{2} .
\end{aligned}
$$

Hence we obtain

$$
\left\|H_{-} f\right\|^{2} \geqslant\|\partial \bar{\partial} f\|^{2}+\left\|(\partial V)^{2} f\right\|^{2}-\left\|\left(\partial^{2} V\right) f\right\|^{2} .
$$

Using (2.41), we can show that

$$
\left\|\left(\partial^{2} V\right) f\right\|^{2} \leqslant a\left\|(\partial V)^{2} f\right\|^{2}+\left(r^{2}+b\right)\|f\|^{2} .
$$

Therefore, we obtain the estimate

$$
\|\partial \bar{\partial} f\|^{2}+(1-a)\left\|(\partial V)^{2} f\right\|^{2} \leqslant\|H f\|^{2}+\left(r^{2}+b\right)\|f\|^{2} .
$$

Since $C_{0}^{\infty}\left(\mathbb{R}^{2} ; \mathbb{C}^{2}\right)$ is a core for $H_{-}$(Proposition 2.1), (2.42) extends to all $f$ in $D\left(H_{-}\right)$, showing at the same time that $D\left(H_{-}\right) \subset D(\partial \bar{\partial}) \cap D\left(|\partial V|^{2}\right)$. Since $H_{-}$is self-adjoint, we conclude that $D\left(H_{-}\right)=D(\partial \bar{\partial}) \cap D\left(|\partial V|^{2}\right)$ and $H_{-}$ $=-\partial \bar{\partial}+|\partial V|^{2}$.

Remark: In the case of polynomial potentials $V$, it is easy to see that (2.41) holds, where $a$ can be made arbitrarily small if $r$ is taken sufficiently large.

Lemma 2.8: Suppose that there exists $r>0$ such that (2.41) holds with $0<a<\frac{1}{2}$ and $b \geqslant 0$. Then $D\left(H_{+}\right)$ $=D(\partial \bar{\partial}) \cap D\left(|\partial V|^{2}\right)$ and (2.22) holds as an operator equality.

Proof: Let 


$$
H_{I}=\left(\begin{array}{cc}
0 & -i\left(\partial^{2} V\right) \\
i\left(\partial^{2} V\right)^{*} & 0
\end{array}\right)
$$

and $f$ be in $D\left(H_{-}\right)$. Then by Lemma 2.7 and the above assumption, we have $D\left(H_{-}\right) \subset D\left(H_{I}\right)$ and

$$
\left\|H_{I} f\right\|^{2} \leqslant a\left\|(\partial V)^{2} f\right\|^{2}+\left(r^{2}+b\right)\|f\|^{2} .
$$

Using (2.42) extended to $f \in D\left(H_{-}\right)$, we obtain

$$
\left\|H_{I} f\right\|^{2} \leqslant \frac{a}{1-a}\left\|H_{-} f\right\|^{2}+\frac{r^{2}+b}{1-a}\|f\|^{2} .
$$

Since $0<a<\frac{1}{2}$, we have $0<a /(1-a)<1$. Therefore, by a standard theorem (the Kato-Rellich theorem) (see, e.g., Ref. 7, Chap. V, Theorem 4.3 and Ref. 8, Theorem X.12), $H_{+}=H_{-}+H_{I}\left[\right.$ see (2.22)] is self-adjoint on $D\left(H_{-}\right)$.

Remark: Polynomial potentials $V$ satisfy the assumption of Lemma 2.8.

Lemma 2.9: Under the same assumption as in Lemma 2.8, we have $D\left(Q_{+}\right)=D(\partial) \cap D(\partial V)$.

Proof: By Lemma 2.8 and its proof, the operators $\overline{\partial \bar{\partial}}$ and $|\partial V|^{2}$ are relatively bounded with respect to $H_{+}$and hence, by a standard theorem (see e.g., Ref. 7, Chap. VI, Theorem I. 38 and Ref. 8, Theorem X.18), relatively form bounded with respect to $H_{+}$. Therefore, in particular, it follows that $D(\partial) \cap D(\partial V) \supset D\left(H_{+}^{1 / 2}\right)=D\left(Q_{+}\right)$. Define the operators

$$
L_{+}=\left(\begin{array}{cc}
-i(\partial V)^{*} & \partial \\
\bar{\partial} & i \partial V
\end{array}\right)
$$

and

$$
L_{-}=\left(\begin{array}{cc}
i \partial V & -\partial \\
-\bar{\partial} & -i(\partial V)^{*}
\end{array}\right)
$$

on $D(\partial) \cap D(\partial V)$. Then the above result shows that

$$
Q_{+} \subset L_{+} .
$$

Obviously, the operator

$$
L \equiv\left(\begin{array}{cc}
0 & L_{-} \\
L_{+} & 0
\end{array}\right)
$$

is a symmetric extension of $Q\lceil D$. Hence we obtain $Q=\bar{L}$ since $Q=\overline{Q \uparrow D}$ is self-adjoint. On the other hand, we have

$$
\bar{L}=\left(\begin{array}{cc}
0 & \bar{L}_{-} \\
\bar{L}_{+} & 0
\end{array}\right) .
$$

By the uniqueness of $Q_{+}$, we obtain $Q_{+}=\bar{L}_{+}$and $Q_{+}^{*}$ $=\bar{L}_{-}$. Hence, by (2.43), we have $Q_{+}=L_{+}=\bar{L}_{+}$. Therefore, $L_{+}$is closed on $D(\partial) \cap D(\partial V)$ and equal to $Q_{+}$.

\section{THE MODEL WITH A SUPERPOTENTIAL OF THE EXPONENTIAL TYPE}

In this section, we consider the model with the potential

$$
V(z)=\lambda e^{\alpha z}, \quad z \in \mathbb{C},
$$

where $\lambda \in \mathbb{C} \backslash\{0\}$ and $\alpha>0$. Then $H_{+}$takes the form

$$
H_{+}=-\partial \bar{\partial}+|\lambda|^{2} \alpha^{2} e^{2 \alpha \operatorname{Re} z}+i \alpha^{2}\left(\begin{array}{cc}
0 & -\lambda e^{\alpha z} \\
\lambda * e^{\alpha z^{*}} & 0
\end{array}\right)
$$

on $D$ [ see (2.22) ], for we have

$$
\partial V(z)=\lambda \alpha e^{\alpha z}, \quad \partial^{2} V(z)=\lambda \alpha^{2} e^{\alpha z} .
$$

For a measurable function $u$ on $[0,2 \alpha]$, we define the functions $f_{u}$ and $\tilde{f}_{u}$ on $\mathbb{R}^{2}$ by

$$
\begin{aligned}
& f_{u}(x, y)=\int_{0}^{2 \alpha} u(p) e^{\alpha x / 2} W_{0,|\alpha-p| / \alpha}\left(4|\lambda| e^{\alpha x}\right) e^{i p y} d p \\
& \tilde{f}_{u}(x, y)=\frac{i}{2 \lambda \alpha} e^{-\alpha(x+i y)}\left(\frac{\partial}{\partial x}+i \frac{\partial}{\partial y}\right) f_{u}(x, y)
\end{aligned}
$$

provided that the right-hand sides are meaningful, where $W_{k, m}(\cdot)$ is the Whittaker function (see e.g., Ref. 9, Chap. XVI). Let

$$
D_{\alpha}=\left\{u \in L^{2}(\mathbb{R}) \mid \operatorname{supp} u \subset(0, \alpha / 2) \cup(\alpha / 2, \alpha)\right\} .
$$

We shall prove the following proposition.

Proposition 3.1: Let $H_{+}$be given by (3.2). Then every vector in $\operatorname{Ker} H_{+}$is of the form $\left(f_{u}, \tilde{f}_{u}\right)$ or $\left(\left(\tilde{f}_{u}\right)^{*}, f_{u}^{*}\right)$, with some $u$ satisfying the condition supp $u \subset[0, \alpha]$ and

Ker $H_{+} \supset\left\{\left(f_{u}, \tilde{f}_{u}\right) \mid u \in D_{\alpha}\right\} \cup\left\{\left(\left(\tilde{f}_{u}\right)^{*}, f_{u}^{*}\right) \mid u \in D_{\alpha}\right\}$.

In particular, $H_{+}$has infinitely many zero-energy states:

$n_{+}=\infty$.

Remarks: (i) The above result shows that in the present model, supersymmetry is unbroken with infinitely degenerate vacua.

(ii) It should be noticed that the Fourier transform $\hat{f}_{u}(x, p)$ [respectively, $\tilde{f}_{u}(x, p)$ ] of $f_{u}(x, y)$ [respectively, $\left.\tilde{f}_{u}(x, y)\right]$ with respect to $y$ has compact support in $p \in \mathbb{R}$. This means physically that every zero-energy state of $H_{+}$is strictly localized in the momentum space dual to the $y$ direction.

(iii) Let

$$
V_{N}(z)=\lambda \sum_{n=0}^{N} \frac{(\alpha z)^{n}}{n !}
$$

and let $Q^{(N)}$ and $H_{+}^{(N)}$ be $Q$ and $H_{+}$with $V=V_{N}$, respectively. Then by Ref. 1, Proposition 9 [see (1.1)], we have $n_{+}(N) \equiv \operatorname{dim} \operatorname{Ker} Q_{+}^{(N)}=\operatorname{dim} \operatorname{Ker} H_{+}^{(N)}=N-1$. On the other hand, it is easy to see that $Q^{(N)}$ and $H_{+}^{(N)}$ converge $Q$ and $H_{+}$in the strong resolvent sense as $N \rightarrow \infty$. Formula (3.8) may be regarded as $n_{+}=\infty=\lim _{N \rightarrow \infty} n_{+}(N)$.

(iv) The corresponding model in the $N=1$ Wess- $\mathrm{Zu}$ mino SSQM (the Witten model ${ }^{2,3}$ ) is given by the Hamiltonian

$H=\left(\begin{array}{cc}H_{+} & 0 \\ 0 & H_{-}\end{array}\right), \quad H_{ \pm}=-\frac{d^{2}}{d x^{2}}+\lambda^{2} \alpha^{2} e^{2 \alpha x} \pm \lambda \alpha^{2} e^{\alpha x}$,

where $\alpha, \lambda \in \mathbb{R} \backslash\{0\}$. This model was discussed in Ref. 10 and it can be shown that $\operatorname{Ker} H_{ \pm}=\{0\} .{ }^{11}$ This result also shows an essential difference between the $N=1$ and $N=2$ Wess-Zumino SSQM's.

Lemma 3.2: For all $r>0$ and all $z \in \mathbb{C}$ with $\left|\partial^{2} V(z)\right| \geqslant r$, the estimate

$$
\left|\partial^{2} V(z)\right| \leqslant\left(\alpha^{2} / r\right)|\partial V(z)|^{2}
$$

holds.

Proof: An elementary exercise.

Lemma 3.3: The operator $H_{+}$is self-adjoint on $D(\partial \bar{\partial}) \cap D\left(|\partial V|^{2}\right)$ and $D\left(Q_{+}\right)=D(\partial) \cap D(\partial V)$.

Proof: By taking $r$ sufficiently large in (3.9), the assumption of Lemma 2.8 is satisfied. Thus Lemmas 2.8 and 2.9 give the desired results.

The following lemma is derived from Lemma 2.6 (i) and (3.3). 
Lemma 3.4: Every vector $(f, g)$ in $\operatorname{Ker} Q_{+}$satisfies

$$
\begin{aligned}
& \left(-\frac{\partial^{2}}{\partial x^{2}}-\frac{\partial^{2}}{\partial y^{2}}+2 \alpha \frac{\partial}{\partial x}\right. \\
& \left.+2 i \alpha \frac{\partial}{\partial y}+4|\lambda|^{2} \alpha^{2} e^{2 \alpha x}\right) f(x, y)=0, \\
& \left(-\frac{\partial^{2}}{\partial x^{2}}-\frac{\partial^{2}}{\partial y^{2}}+2 \alpha \frac{\partial}{\partial x}\right. \\
& \left.-2 i \alpha \frac{\partial}{\partial y}+4|\lambda|^{2} \alpha^{2} e^{2 \alpha x}\right) g(x, y)=0,
\end{aligned}
$$

where we put $z=x+i y$.

Lemma 3.5: Let $a \in \mathbb{R}, b>0, c>0$, and $E \in \mathbb{C}$ be constants.

(i) If $\operatorname{Re} E \leqslant 0$, then

$$
\left(-\frac{d^{2}}{d x^{2}}+2 a \frac{d}{d x}+c e^{2 b x}\right) f(x)=E f(x)
$$

has no solutions $f \neq 0$ with $f \in D\left(d^{2} / d x^{2}\right) \cap D\left(e^{2 b x}\right)$ $\subset L^{2}(\mathbb{R})$.

(ii) Suppose that

$$
0 \leqslant\left|\operatorname{Re} \sqrt{a^{2}-E}\right|<a, \quad \operatorname{Re} E>0
$$

and $2 \sqrt{a^{2}-E} / b$ is not an integer.

Then every nonzero solution $f \in L^{2}(\mathbb{R})$ to Eq. (3.12) is given by

$$
f(x)=K e^{(a-b / 2) x} W_{0, \sqrt{a^{2}-E / b}}\left(2 \sqrt{c} e^{b x} / b\right),
$$

with a constant $K \in \mathbb{C} \backslash\{0\}$, where $W_{k, m}(z)$ is the Whittaker function (see e.g., Ref. 9, Chap. XVI).

Proof: (i) Via the elliptic regularity, every solution $f$ to Eq. (3.12) is $C^{\infty}$. Let $f \in D\left(d^{2} / d x^{2}\right) \cap D\left(e^{2 b x}\right)$ be a nonzero solution to Eq. (3.12). Taking the inner product of $f$ with (3.12) in $L^{2}(\mathbb{R})$, we obtain

$$
\left\|f^{\prime}\right\|^{2}+2 a\left(f^{\prime}, f\right)+c\left\|e^{b x} f\right\|^{2}=E\|f\|^{2} \text {. }
$$

Since $\left(f^{\prime}, f\right)$ is pure imaginary, it follows that $\operatorname{Re} E>0$. Thus the desired result follows.

(ii) Let $f \in L^{2}(\mathbb{R})$ be a nonzero solution to Eq. (3.12) and define

$v(t)=t^{(b-2 a) / 2 b} f\left(1 / 2 b \log \left(b^{2} t^{2} / 4 c\right)\right), \quad t>0$.

Then we have

$$
\|f\|_{2}^{2}=\frac{1}{b} \int_{0}^{\infty} t^{2(a-b) / b}|v(t)|^{2} d t<\infty
$$

and $v$ satisfies the Whittaker equation

$$
\frac{d^{2}}{d t^{2}} v(t)+\left\{-\frac{1}{4}+\frac{\frac{1}{4}-\left(a^{2}-E\right) / b^{2}}{t^{2}}\right\} v(t)=0 \text {. }
$$

Since $2 \sqrt{a^{2}-E} / b$ is not an integer by assumption, the confluent hypergeometric functions $M_{0, \sqrt{a^{2}-E / b}}(t)$ and $M_{0,-\sqrt{ } a^{2}-E / b}(t)$ (see, e.g., Ref. 9, Chap. XVI) form a fundamental system of solutions to (3.16); every solution to (3.16) is given by a linear combination of these functions. By taking the asymptotic property of $M_{0, \mu}(t)$ as $t \rightarrow 0$ and $t \rightarrow \infty$ into account, we see that possible solutions to (3.16) with condition (3.15) are scalar multiples of the Whittaker function $W_{0, \sqrt{a^{2}}-E / b}(t)$, with

$$
0 \leqslant\left|\operatorname{Re} \sqrt{a^{2}-E}\right|<a .
$$

Condition (3.17) comes from the integrability condition of $t^{2(a-b) / b}|v(t)|^{2}$ near $t=0$. On the contrary, if we define $f(x)$ by relation (3.14) with $v(t)=W_{0, \sqrt{a^{2}}-E / b}(t)$ under condition (3.15), then $f$ is in $L^{2}(\mathbb{R})(f \neq 0)$ and satisfies Eq. (3.12).

Lemma 3.6: (i) Every solution $f \in D(\overline{\partial \bar{\partial}}) \cap D\left(e^{2 \alpha x}\right)$ to Eq. (3.10) has the form (3.4).

(ii) For all $u \in D_{\alpha}$, the function $f_{u}$ given by (3.4) is a solution to Eq. (3.10) with $f_{u} \in D(\overrightarrow{\partial \partial}) \cap D\left(e^{2 \alpha x}\right)$.

Remark: The sets of the solutions $g$ of Eq. (3.11) consist of the complex conjugates of the solutions $f$ to (3.10).

Proof: (i) If $f$ is in $D(\overline{\partial \partial}) \cap D\left(e^{2 \alpha x}\right)$, then Eq. (3.10) is equivalent to

$\left(-\frac{\partial^{2}}{\partial x^{2}}+2 \alpha \frac{\partial}{\partial x}+4|\lambda|^{2} \alpha^{2} e^{2 \alpha x}\right) \hat{f}(x, p)=E(p) \hat{f}(x, p)$,

with

$$
E(p)=p(2 \alpha-p), \quad p \in \mathbb{R},
$$

where $\hat{f}(x, p)$ is the Fourier transform of $f(x, y)$ with respect to $y$ :

$$
\hat{f}(x, p)=\frac{1}{\sqrt{2 \pi}} \int e^{-i p y} f(x, y) d y .
$$

Via Lemma 3.5 (i), Eq. (3.18) has no nonzero solutions $\hat{f}(\cdot, p) \in D\left(d^{2} / d x^{2}\right) \cap D\left(e^{2 \alpha x}\right)$ if $E(p) \leqslant 0$. Hence $\hat{f}(\cdot, p)=0$ for all $p \notin(0,2 \alpha)$.

Let $E(p)>0$, i.e., $0<p<2 \alpha$. Then by Lemma 3.5 (ii), every solution $\hat{f}(\cdot, p) \in L^{2}(\mathbb{R})$ to Eq. (3.18) is given by

$$
\hat{f}(x, p)=u(p) e^{a x / 2} W_{0,|\alpha-p| / \alpha}\left(4|\lambda| e^{\alpha x}\right)=\hat{f}_{u}(x, p),
$$

where $u$ is a function on the set $S \equiv\{p \in \mathbb{R} \mid 0<p<2 \alpha, p \neq \alpha$ / $\left.2, \alpha, \frac{3}{2} \alpha\right\}$. Thus the desired result follows.

(ii) Let $u \in D_{\alpha}$. We need only show that $f_{u}$ is in $D(\overline{\partial \partial}) \cap D\left(e^{2 \alpha x}\right)$ : We write it as $f_{u}=f$. By the asymptotics of the Whittaker function $W_{0, \mu}(t)$ at $t=0$ and $t=+\infty$ (see, e.g., Ref. 9, Chap. XVI), we see that $\hat{f}$ and $e^{2 \alpha x} \hat{f}(x, p)$ are in $L^{2}\left(\mathbb{R}^{2}\right)$. By using the recursion relation

$$
z W_{0, m}^{\prime}(z)=(z / 2) W_{0, m}(z)-W_{1, m}(z),
$$

we can show that

$$
\begin{aligned}
\frac{\partial}{\partial x} \hat{f}(x, p)= & \frac{\alpha}{2} \hat{f}(x, p)+2|\lambda| \alpha e^{\alpha x} \hat{f}(x, p) \\
& -\alpha u(p) e^{\alpha x / 2} W_{1,|\alpha-p| / \alpha}\left(4|\lambda| e^{\alpha x}\right)
\end{aligned}
$$

Each term on the rhs of $(3.20)$ is in $L^{2}\left(\mathbb{R}^{2}\right)$ and hence $\hat{\partial f}(x, p) / \partial x$ is in $L^{2}\left(\mathbb{R}^{2}\right)$. By virtue of (3.18), this implies that $\partial^{2} \hat{f}(x, p) / \partial x^{2}$ is in $L^{2}\left(\mathbb{R}^{2}\right)$. We can also see that $p^{2} \hat{f}(x, p)$ is in $L^{2}\left(\mathbb{R}^{2}\right)$. Thus the function $f$ is in $D(\overline{\partial \bar{\partial}}) \cap D\left(e^{2 \alpha x}\right)$.

Lemma 3. 7: Let $f_{u}$ be given by (3.4) with $u \in D_{\alpha}$. Then $f_{u}$ is in $D(\partial) \cap D\left(e^{\alpha x}\right)$ and $e^{-\alpha x} \partial f_{u}$ is in $L^{2}\left(\mathbb{R}^{2}\right)$.

Proof: By the proof of Lemma 3.6 we have $f_{u}$ $\in D(\overline{\partial \bar{\partial}}) \cap D\left(e^{2 \alpha x}\right) . \quad$ Since $\quad D(\overline{\partial \bar{\partial}}) \cap D\left(e^{2 \alpha x}\right) \subset D(\partial)$ $\cap D\left(e^{\alpha x}\right)$, we obtain $f_{u} \in D(\partial) \cap D\left(e^{\alpha x}\right)$.

$$
\begin{aligned}
& \text { Let } \\
& h(x, y)=e^{-\alpha x}\left(\bar{\partial} f_{u}\right)(x, y) .
\end{aligned}
$$

Then we have

$$
\hat{h}(x, p)=\frac{1}{2} e^{-\alpha x}\left(\frac{\partial}{\partial x} \hat{f}_{u}(x, p)-p \hat{f}_{u}(x, p)\right) .
$$


By using (3.20), we obtain

$$
2 \hat{h}(x, p)=R(x, p)+2|\lambda| \alpha \hat{f}_{u}(x, p),
$$

where

$$
\begin{aligned}
R(x, p)= & u(p) e^{-\alpha x / 2}\left\{(\alpha / 2-p) W_{0,|\alpha-p| / \alpha}\left(4|\lambda| e^{\alpha x}\right)\right. \\
& \left.-\alpha W_{1,|\alpha-p| / \alpha}\left(4|\lambda| e^{\alpha x}\right)\right\} .
\end{aligned}
$$

Since $\hat{f}_{u}$ is in $L^{2}\left(\mathbb{R}^{2}\right)$, we need only show that the function $R$ is in $L^{2}\left(\mathbb{R}^{2}\right)$. It is easy to see that for every $c \in \mathbb{R}$,

$$
\int_{\mathbb{R}} d p \int_{c}^{\infty} d x|R(x, p)|^{2}<\infty .
$$

By the asymptotics of $W_{k, m}(z)$ at $z=0$ and by virtue of the condition supp $u \subset(0, \alpha / 2) \cup(\alpha / 2, \alpha)$, we see that

$$
\begin{aligned}
e^{\alpha x / 2} R(x, p) \underset{x \rightarrow-\infty}{\sim} & u(p) w_{1}(p) \\
& \times\left(4|\lambda| e^{\alpha x}\right)^{-|\alpha-p| / \alpha+1 / 2} \cdot\left(4|\lambda| e^{\alpha x}\right)
\end{aligned}
$$

and hence

$$
R(x, p) \underset{x \rightarrow-\infty}{\sim} u(p) w_{2}(p) e^{(\alpha-|\alpha-p|) x},
$$

with the continuous functions $w_{1}$ and $w_{2}$ on the support of $u$. Therefore, we have

$$
\int_{\mathbb{R}} d p \int_{-\infty}^{c} d x|R(x, p)|^{2}<\infty .
$$

Thus we obtain $R \in L^{2}\left(\mathbb{R}^{2}\right)$.

Proof of Proposition 3.1: Via Lemma 3.6, the set of solutions to Eq. (3.10) in $D(\overrightarrow{\partial \partial}) \cap D\left(e^{2 \alpha x}\right)$ consist of just functions of the form $f_{u}$ given by (3.4). If $f_{u}$ and $\tilde{f}_{u}$ given by (3.4) and (3.5), respectively, are in $L^{2}\left(\mathbb{R}^{2}\right)$, then $R(\cdot, p)$ defined by (3.21) must be in $L^{2}(\mathbb{R})$ for a.e. $p \in[0,2 \alpha]$. We can show that if $\alpha \leqslant p \leqslant 2 \alpha$, then $R(\cdot, p)$ is not in $L^{2}(\mathbb{R})$ (cf. the proof of Lemma 3.7). Hence we have supp $u \subset[0, \alpha]$. Then the first assertion follows from Lemma 2.6 (ii) and (iii) and the remark after Lemma 3.6.

Via Lemma 3.7, every $f_{u}$ with $u \in D_{\alpha}$ satisfies the assumption of Lemma 2.6(ii). Therefore, for every $u \in D_{\alpha}$, the pair $\left(f_{u}, \tilde{f}_{u}\right)$, with $\tilde{f}_{u}$ given by $(3.5)$, is in $\operatorname{Ker} Q_{+}$ $=$ Ker $H_{+}$. Via Lemma 2.6 (iii) and the remark after Lemma 3.6 , we also have $\left(\left(\tilde{f}_{u}\right)^{*}, f_{u}^{*}\right) \in \operatorname{Ker} H_{+}$for every $u \in D_{\alpha}$. Thus we obtain (3.7). We have $\operatorname{dim} D_{\alpha}=\infty$ and, if the vectors $u_{1}, \ldots, u_{n} \in D_{\alpha}$ are linearly independent, then so are the vectors $\left(f_{u_{1}}, \tilde{f}_{u_{1}}\right), \ldots,\left(f_{u_{n}}, \tilde{f}_{u_{n}}\right)$. Thus (3.8) follows.

\section{APPLICATION}

In this section, we apply the result in Sec. III to models in atomic and nuclear physics, which were discussed in Ref. 1.

\section{A. Nonrelativistic spin- $\frac{1}{2}$ particle in an external SU(2) gauge field}

Let $\tau_{a}, a=1,2,3$ be the generators of the $\mathrm{SU}(2)$ group. An external $\mathrm{SU}(2)$ gauge field $A(x)=\left(A_{1}(x), A_{2}(x)\right.$, $\left.A_{3}(x)\right), \quad x=\left(x_{1}, x_{2}, x_{3}\right) \in \mathbb{R}^{3} \quad$ is given as $A_{j}(x)$ $=\Sigma_{a=1}^{3} A_{j}^{a}(x) \tau_{a}, j=1,2,3$, where we take $A_{j}^{a}$ to be real valued. Then the Hamiltonian of a nonrelativistic spin- $\frac{1}{2}$ particle with mass 1 coupled minimally to the gauge field is given by

$$
H_{A}=\frac{1}{2}(-i \nabla-g A)^{2}-\frac{1}{2} \sigma \cdot B,
$$

where $g \in \mathbb{R} \backslash\{0\}$ is a coupling constant, $\nabla=\left(\partial / \partial x_{1}, \partial /\right.$ $\left.\partial x_{2}, \partial / \partial x_{3}\right), \quad \sigma=\left(\sigma_{1}, \sigma_{2}, \sigma_{3}\right), \quad$ and $\quad B_{j}=g(\operatorname{curl} A)_{j}$ $+\frac{1}{2} g^{2} \Sigma_{k, m=1}^{3} \epsilon_{j k m}\left[A_{k}, A_{m}\right]$ (where $\epsilon_{j k m}$ is the Kronecker antisymmetric symbol).

We consider the following situation.

(a): (i) $A_{1}=A_{2}=0$, (ii) $A_{3}^{3}=0$, (iii) $A_{3}^{1}$ (x) and $A_{3}^{2}(x)$ depend only on $x_{1}$ and $x_{2}$, and (iv) $\nabla_{3}=0$, where condition (iv) means that we consider only state vectors independent of $x_{3}$ and identify them with elements in $L^{2}\left(\mathbb{R}^{2} ; \mathbb{C}^{4}\right)$. Let $V$ be a holomorphic function on $\mathbb{C}$ and $H$ be given by (2.21). Then it was shown in Ref. 1 that under condition (a), with $A_{3}^{1}-i A_{3}^{2}=2 \partial V / g, H_{A}$ can be written as

$$
H_{A}=2 H
$$

with a rearrangement of components.

Proposition 4.1: Suppose that condition (a) is satisfied. Let

$$
\begin{aligned}
& A_{3}^{1}\left(x_{1}, x_{2}\right)=(2 \alpha \lambda / g) e^{\alpha x_{1}} \cos \alpha x_{2}, \\
& A_{3}^{2}\left(x_{1}, x_{2}\right)=-(2 \alpha \lambda / g) e^{\alpha x_{1}} \sin \alpha x_{2},
\end{aligned}
$$

with $\alpha>0$ and $\lambda \in \mathbb{R} \backslash\{0\}$. Then $H_{A}$ has infinitely many zeroenergy states $\Psi$ in $L^{2}\left(\mathbb{R}^{2} ; \mathbb{C}^{4}\right): H_{A} \Psi=0$. Further, the Fourier transform $\hat{\Psi}\left(x_{1}, p\right)$ of every $\Psi\left(x_{1}, x_{2}\right)$ with respect to $x$, has compact support in $p \in \mathbb{R}$.

Proof: By (4.3) and (4.4), we have

$$
A_{3}^{1}-i A_{3}^{2}=2 \frac{\partial V}{g},
$$

with $V(z)=\lambda e^{\alpha z}\left(z=x_{1}+i x_{2}\right)$. Thus by (4.2) and Proposition 3.1, we obtain the desired result.

Remark: The gauge field given by (4.3) and (4.4) is periodic in the $x_{2}$ direction. This may be an origin of the existence of infinitely many zero-energy states and the localization of the states in the momentum space dual to the $x_{2}$ direction.

\section{B. Nonrelativistic nucleon in an external pion field}

The Hamiltonian of a nonrelativistic nucleon in an external pion field $\phi(x)=\left\{\phi_{1}(x), \phi_{2}(x), \phi_{3}(x)\right), x \in \mathbb{R}^{3}$ is given by

$$
H_{\phi}=-\frac{1}{2} \nabla^{2}+\frac{1}{2} g \sigma \cdot \nabla(\tau \cdot \phi(x))+\frac{1}{2} g^{2} \phi(x)^{2} .
$$

(See Ref. 1.) Suppose that the following condition is satisfied.

Condition ( $\phi$ ): (i) $\phi_{3}=0$, (ii) $\phi_{1}$ and $\phi_{2}$ depend only on $x_{1}$ and $x_{2}$, and (iii) $\nabla_{3}=0$.

Then it was shown in Ref. 1 that

$H_{\phi}=2 H$,

with $\phi_{1}-i \phi_{2}=-2 i \partial \mathrm{V} / \mathrm{g}$.

Proposition 4.2: Suppose that condition $(\phi)$ is satisfied Let

$$
\begin{aligned}
& \phi_{1}\left(x_{1}, x_{2}\right)=(2 \lambda \alpha / g) e^{\alpha x_{1}} \sin \alpha x_{2}, \\
& \phi_{2}\left(x_{1}, x_{2}\right)=(2 \lambda \alpha / g) e^{\alpha x_{1}} \cos \alpha x_{2},
\end{aligned}
$$

with $\alpha>0$ and $\lambda \in \mathbb{R} \backslash\{0\}$. Then, $H_{\phi}$ has infinitely many zero-energy states $\Phi$ in $L^{2}\left(\mathbb{R}^{2} ; \mathbb{C}^{4}\right): H_{\phi} \Phi=0$. Further, the Fourier transform $\hat{\Phi}\left(x_{1}, p\right)$ of every $\Phi\left(x_{1}, x_{2}\right)$ with respect to $x_{2}$ has compact support in $p \in \mathbb{R}$.

Proof: Similar to the proof of Proposition 4.1. 


\section{ACKNOWLEDGMENTS}

This research was supported in part by Grant-In-Aid Nos. 63740063 and 62460001 for science research from the Ministry of Education, Japan.

\section{APPENDIX: KERNEL OF A SCHRÖDINGER OPERATOR}

Let $d$ be an arbitrarily fixed positive integer. Let $U$ be a real-valued measurable function on $\mathbb{R}^{d}$ and $\Delta$ be the $d$-dimensional Laplacian: $\Delta=\Sigma_{j=1}^{d} \partial^{2} / \partial x_{j}^{2}, \quad x=\left(x_{1}, \ldots, x_{d}\right)$ $\in \mathbb{R}^{d}$. We consider the Schrödinger operator

$$
H_{\mathrm{s}}=-\Delta+U
$$

acting in $L^{2}\left(\mathbb{R}^{d}\right)$.

Lemma Al: Suppose that $C_{0}^{2}\left(\mathbb{R}^{d}\right) \subset D(U)$ and $U \geqslant 0$. Let $\bar{H}_{\mathrm{S}}$ be the closure of $H_{\mathrm{S}} \backslash C_{0}^{2}\left(\mathbb{R}^{2}\right)$. Then we have

$\operatorname{Ker} \bar{H}_{\mathrm{s}}=\{0\}$.

Remark: (i) It is obvious that under the condition $C_{0}^{2}\left(\mathbb{R}^{d}\right) \subset D(U), H_{\mathrm{s}} \backslash C_{0}^{2}\left(\mathbb{R}^{d}\right)$ is closable and symmetric.

(ii) The domain $D\left(\bar{H}_{\mathrm{s}}\right)$ is not necessarily equal to $D(\Delta) \cap D(U)$. This is the reason why we need a limiting argument to prove (A2) (see below).

Proof: Let $f \in \operatorname{Ker} \bar{H}_{\mathrm{S}}: \bar{H}_{\mathrm{S}} f=0$. Then we can take a sequence $\left\{f_{n}\right\} \subset C_{0}^{2}\left(\mathbb{R}^{d}\right)$ such that $f_{n} \stackrel{s}{\rightarrow} f$ and $H_{\mathrm{S}} f_{n} \stackrel{s}{\rightarrow}$ $0(n \rightarrow \infty)$ : It follows from the latter that

$$
\sum_{j=1}^{d}\left\|D_{j} f_{n}\right\|^{2}+\left\|U^{1 / 2} f_{n}\right\|^{2} \rightarrow 0,
$$

where $D_{j}=\partial / \partial x_{j}$. Hence we have $D_{j} f_{n} \stackrel{s}{\rightarrow} 0, j=1, \ldots, d$, and
$U^{1 / 2} f_{n} \stackrel{s}{\rightarrow} 0$. Since $D_{j}, j=1, \ldots, d$, are closed, it follows that $f \in D\left(D_{j}\right), j=1, \ldots, d$, and

$$
D_{j} f=0, \quad j=1, \ldots, d,
$$

which, together with $f \in L^{2}\left(\mathbb{R}^{d}\right)$, imply that $f=0$.

\footnotetext{
'A. Jaffe, A. Lesniewski, and M. Lewenstein, "Ground state structure in supersymmetric quantum mechanics," Ann. Phys. 178, 313 (1987).

${ }^{2}$ E. Witten, "Dynamical breaking of supersymmetry," Nucl. Phys. B 185, $513(1981)$.

${ }^{3}$ E. Witten, "Constraints on supersymmetry breaking," Nucl. Phys. B 202, 253 (1982).

${ }^{4} \mathrm{P}$. Chernoff, "Essential self-adjointness of powers of generators of hyperbolic equations," J. Funct. Anal. 12, 401 (1973).

${ }^{5}$ A. Arai, "Supersymmetry and singular perturbations,"J. Funct. Anal. 60, 378 (1985).

${ }^{6} \mathrm{H}$. Grosse and L. Pittner, "Supersymmetric quantum mechanics defined as sesquilinear forms," J. Phys. A Math. Gen. 20, 4265 (1987).

'T. Kato, Perturbation Theory for Linear Operators (Springer, Berlin, 1976), 2nd ed.

${ }^{8} \mathrm{M}$. Reed and B. Simon, Methods of Modern Mathematical Physics Vol. II: Fourier Analysis, Self-Adjointness (Academic, New York, 1975).

${ }^{9} \mathrm{E}$. T. Whittaker and G. N. Watson, A Course of Modern Analysis (Cambridge U. P., London, 1969), 4th ed.

1"R. Akhoury and A. Comtet, "Anomalous behavior of the Witten indexExactly soluble models," Nucl. Phys. B 246, 253 (1984).

"In Ref. 10, the Witten index $I_{\mathrm{w}}$ is computed formally and the two different results $I_{\mathrm{w}}=-\frac{1}{2}$ and $I_{\mathrm{w}}=0$ are derived according to the two different methods of calculation. We can prove rigorously that $\operatorname{Ker} H_{ \pm}=\{0\}$ and hence $I_{\mathrm{w}}=0$. The method of the proof is similar to that in the present paper.
} 
Journal of Mathematical Physics is copyrighted by the American Institute of Physics (AIP). Redistribution of journal material is subject to the AIP online journal license and/or AIP copyright. For more information, see http://ojps.aip.org/jmp/jmpcr.jsp Copyright of Journal of Mathematical Physics is the property of American Institute of Physics and its content may not be copied or emailed to multiple sites or posted to a listserv without the copyright holder's express written permission. However, users may print, download, or email articles for individual use. 